\title{
Acute disseminated encephalomyelitis in a COVID-19 pediatric patient
}

\author{
Adélia Maria de Miranda Henriques-Souza ${ }^{1}$ (D) Ana Cláudia Marques Gouveia de Melo ${ }^{1}$ (D) \\ Bianca de Aguiar Coelho Silva Madeiro ${ }^{2}$ (D) - Leonardo Furtado Freitas ${ }^{4}$ (D) - Pedro Augusto Sampaio Rocha-Filho ${ }^{2,3}$ (D) \\ Fabrício Guimarães Gonçalves ${ }^{5}$ (i)
}

Received: 29 June 2020 / Accepted: 23 September 2020 / Published online: 1 October 2020

(C) Springer-Verlag GmbH Germany, part of Springer Nature 2020

\begin{abstract}
The authors present a case of acute disseminated encephalomyelitis in a COVID-19 pediatric patient with positive SARS-CoV2 markers from a nasopharyngeal swab. A previously healthy 12-year-old-girl presented with a skin rash, headache, and fever. Five days after that, she had an acute, progressive, bilateral, and symmetrical motor weakness. She evolved to respiratory failure. Magnetic resonance imaging (MRI) of the brain and cervical spine showed extensive bilateral and symmetric restricted diffusion involving the subcortical and deep white matter, a focal hyperintense T2/ FLAIR lesion in the splenium of the corpus callosum with restricted diffusion, and extensive cervical myelopathy involving both white and gray matter. Follow-up examinations of the brain and spine were performed 30 days after the first MRI examination. The images of the brain demonstrated mild dilatation of the lateral ventricles and widespread widening of the cerebral sulci, complete resolution of the extensive white matter restricted diffusion, and complete resolution of the restricted diffusion in the lesion of the splenium of the corpus callosum, leaving behind a small gliotic focus. The follow-up examination of the spine demonstrated nearly complete resolution of the extensive signal changes in the spinal cord, leaving behind scattered signal changes in keeping with gliosis. She evolved with partial clinical and neurological improvement and was subsequently discharged.
\end{abstract}

Keywords COVID-19 $\cdot$ Brain diseases $\cdot$ Spinal cord diseases $\cdot$ ADEM $\cdot$ Child $\cdot$ Central Nervous System

\section{Introduction}

The majority of COVID-19 pediatric patients are asymptomatic [1]. In the pediatric age group, very few reports of neurological manifestations are available [2, 3]. Multiple COVID-19-related neurological manifestations have been described in adults, such

Adélia Maria de Miranda Henriques-Souza adelianeuro@gmail.com

Ana Cláudia Marques Gouveia de Melo anagouveianeuro@yahoo.com

Bianca de Aguiar Coelho Silva Madeiro bianca.madeiro@upe.br

Leonardo Furtado Freitas furtadoleofreitas@hotmail.com

Pedro Augusto Sampaio Rocha-Filho pedroasampaio@gmail.com as anosmia, ageusia, headache, encephalopathy, acute hemorrhagic necrotizing encephalopathy, meningitis, Guillain-Barre syndrome, stroke, myopathy, and acute disseminated encephalomyelitis (ADEM) [4-7]. Neurologic damage may occur by direct virus lesion, cytokine-related injury, and secondary hypoxia $[7,8]$. We report a case of acute disseminated

Fabrício Guimarães Gonçalves goncalves.neuroradio@gmail.com

1 Hospital da Restauração, Recife, Brazil

2 Hospital Universitário Oswaldo Cruz, Universidade de Pernambuco (UPE), Recife, Brazil

3 Department of Neuropsychiatry, Universidade Federal de Pernambuco (UFPE), Recife, Brazil

4 Hospital Beneficência Portuguesa - BP Medicina Diagnóstica, São Paulo, Brazil

5 Department of Radiology, Division of Neuroradiology, Children's Hospital of Philadelphia, Philadelphia 19104, PA, USA 
encephalomyelitis in a COVID-19 pediatric patient, with positive SARS-CoV2 markers from a nasopharyngeal swab.

\section{Case presentation}

A previously healthy 12 -year-old girl presented with a skin rash, headache, and fever $\left(38^{\circ} \mathrm{C}\right)$. Headache and fever lasted 1 day, and the skin rash lasted 6 days. Five days after the onset of symptoms, she had an acute, progressive, bilateral, and symmetrical motor weakness (inability to stand, walk, and handle objects). In addition, she had tingling and numbness in the inferior limbs but no sphincter abnormalities. Up to this point, she had had no respiratory symptoms. Two days after the onset of the neurological symptoms, she developed respiratory distress (venturi mask at 50\%; $\mathrm{PCO}_{2}: 120 \mathrm{mmHg} ; \mathrm{PaO}_{2}$ $108 \mathrm{mmHg}$ ) that evolved to respiratory failure. She was intubated and placed on mechanical ventilation.

She was unable to follow commands. Her oculocephalic, corneal, cough, and gag reflexes were abolished but had photoreactive isochoric pupils. She also had flaccid tetraplegia, deep areflexia, and abolished abdominal cutaneous and plantar cutaneous reflexes. There was no history of cardiorespiratory arrest or visual disturbances.

White cell count was notable for leukocytosis with neutrophilia $\left(13.87 \times 10^{3}\right.$ white blood cells $\left./ \mu \mathrm{L}\right)$. Red blood cells, electrolytes, urea, and creatinine were normal. Her creatine phosphokinase was $556 \mathrm{U} / \mathrm{L}$; aspartate aminotransferase, $49 \mathrm{U} / \mathrm{L}$; alanine aminotransferase, $104 \mathrm{U} / \mathrm{L}$; lactate dehydrogenase, $373 \mathrm{U} / \mathrm{L}$; C-reactive protein, $4.1 \mathrm{mg} / \mathrm{dL}$; fibrinogen, $461 \mathrm{mg} / \mathrm{dL}$; D-dimer, $6.470 \mathrm{ng} / \mathrm{mL}$; and troponin, 5.5. SARSCoV-2 real-time reverse transcriptase (rRT)-PCR from a nasopharyngeal swab, collected on the sixth day of symptoms, was positive (XGEN MASTER COVID-19-CDC China protocol-ORF1ab and N SARS-CoV-2 gene targets). CSF analysis demonstrated $18 \mathrm{mg} / \mathrm{dL}$ of protein, $74 \mathrm{mg} / \mathrm{dL}$ of glucose, no cells, and normal opening pressure. (rRT)-PCR for SARS-CoV-2, Zika virus, and dengue viruses serotypes 1 to 4 (DENV-1-4) were negative in the CSF.

Low-dose computed tomography (LDCT) of the lungs demonstrated no ground glass changes, but a right lower lobe focal atelectasis, that resolved after 3 days of respiratory therapy. LDCT of the lungs findings were not in keeping with COVID-19-related changes and interpreted as due to airway hypersecretion and mucous plug.

Magnetic resonance imaging (MRI) of the brain, acquired on the seventh day after the fever and on the second day after the onset of the neurological symptoms, showed extensive bilateral and symmetric restricted diffusion involving the subcortical and deep white matter (Fig. 1a and b). There was also a focal hyperintense T2/fluid-attenuated inversion recovery (FLAIR) lesion in the splenium of the corpus callosum (Fig. $1 \mathrm{c}$ and d) with restricted diffusion. No signs of blood-brain barrier breakdown were identified in the contrast-enhanced T1-weighted images. Susceptibility-weighted images were obtained, which did not demonstrate hemorrhagic deposits or pathological calcification in the brain parenchyma. MRI of the cervical spine showed longitudinally extensive cervical myelopathy involving both white and gray matter (Fig. 2a and b). Diffusion-weighted images were not acquired, nor gadolinium that was injected in the spine examination.

She was treated clinically under the institutional protocol, including a 5-day therapy with methylprednisolone, which was repeated due to little improvement of her weakness after first pulse therapy. She remained on mechanical ventilation for 20 days. Her cognition was spared, as was her cranial nerve functions (except for mild dysphagia and dysphonia).

A follow-up examination of the brain was performed 30 days after the first MRI examination, which demonstrated mild dilatation of the lateral ventricles, widespread widening of the cerebral sulci, complete resolution of the extensive white matter restricted diffusion, and complete resolution of the restricted diffusion in the lesion of the splenium of the corpus callosum, with a residual small gliotic focus. A follow-up examination of the spine demonstrated nearly complete resolution of the extensive signal changes in the spinal cord, with residual scattered signal changes in keeping with gliosis.

Sixty-eight days after the onset of the condition, she had evolved from flaccid to spastic tetraparesis. Her strength improved from grade zero in the four limbs to grade three in the left superior limb, grade three in the lower limbs, and grade two in the right superior limb, using the Medical Research Council Scale (MRC) for muscle strength. She regained cervical control, being able to sit with support and reach objects placed nearby. Global hyperreflexia and bilateral Babinski signs were present. She had incomplete sphincter control. A second SARS-CoV-2 (rRT)-PCR from a nasopharyngeal swab was obtained 60 days after the onset of symptoms (before hospital discharge), which was negative.

\section{Discussion}

The striking neuroimaging features, in this case, are the diffuse subcortical and deep white matter-restricted diffusion, focal T2/FLAIR hyperintense lesions in the splenium of the corpus callosum, and inferior medulla, and extensive cervical myelopathy. The presence of acute and diffuse encephalomyelitis in a pediatric patient (mainly involving the cerebral white matter) following a viral infection due to SARS-CoV-2 favors the diagnosis of ADEM.

According to the consensus criteria established by the International Pediatric Multiple Sclerosis Study Group in 2013 [9], the diagnosis of pediatric ADEM requires all of the following: (1) a polyfocal, clinical central nervous system (CNS) event with a presumed inflammatory demyelinating 
Fig. 1 Axial diffusion (a) and apparent diffusion coefficient map images (b) showing extensive bilateral and symmetric restricted diffusion involving the subcortical and deep white matter. Axial T2 (c), fluid-attenuated inversion recovery (FLAIR) (d), and ADC map images (e) showing a focal hyperintense lesion in the splenium of the corpus callosum with restricted diffusion (arrows)
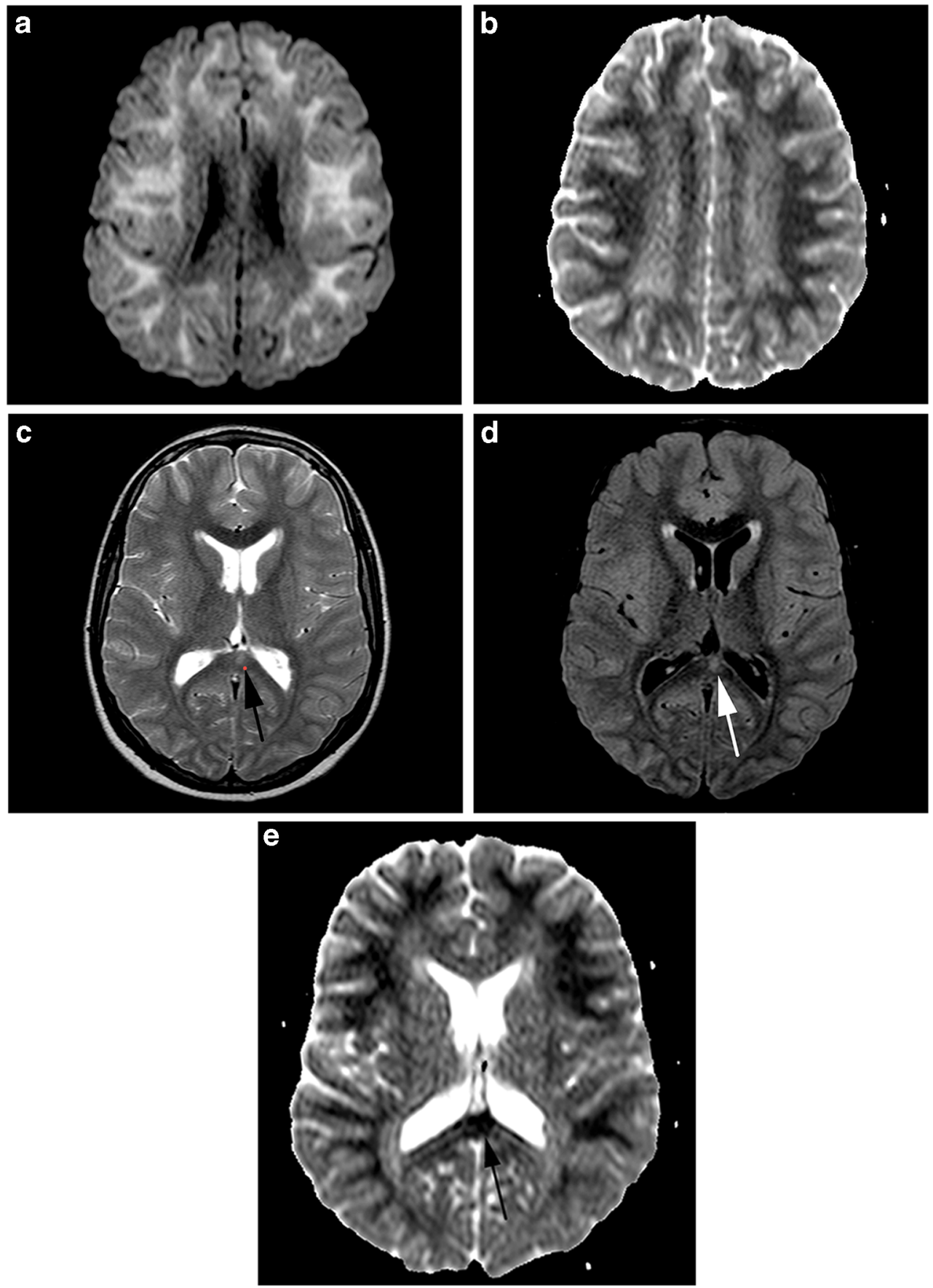

cause; (2) an encephalopathy that cannot be explained by fever; (3) no new clinical and MRI findings emerging 3 months or more after the onset; (4) abnormal brain MRI during the acute phase. Typical MRI findings include (a) diffuse, poorly demarcated, large $(>1-2 \mathrm{~cm})$ lesions involving predominantly the cerebral white matter; (b) rare white matter T1 hypointense lesions; and (c) possible deep gray matter lesions (e.g., thalamus or basal ganglia).

Five distinct patterns of cerebral involvement have been described to classify the MRI findings in children with
ADEM: (1) ADEM with small lesions (less than $5 \mathrm{~mm}$ ); (2) ADEM with large, confluent, or tumefactive lesions, with frequent extensive perilesional edema and mass effect; (3) ADEM with additional symmetrical bithalamic involvement; (4) acute hemorrhagic encephalomyelitis (AHEM), when the blood products are identified within the large T2 hyperintense demyelinating lesions; and (5) ADEM with a pseudoleukodystrophy pattern, with a diffuse, bilateral, symmetrical, and usually nonenhancing white matter involvement [10-13]. We believe that the ADEM pattern that fits our patient is the 
Fig. 2 Sagittal (a) and axial T2weighted images (b) highlighting longitudinally extensive cervical myelopathy involving both white and gray matter
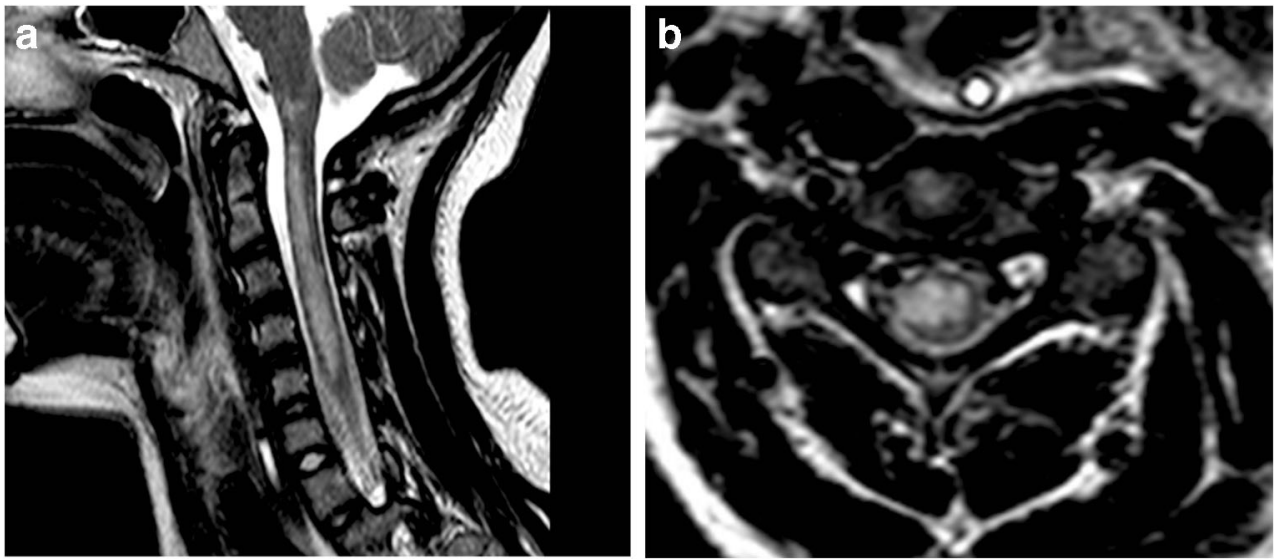

one that courses with diffuse, bilateral, symmetrical, and nonenhancing white matter involvement (ADEM with pseudoleukodystrophy pattern).

Well-known entities associated with involvement of the splenium of the corpus callosum include the reversible splenial lesion syndrome (RESLES) and MERS, which is a clinical-radiological entity characterized by mild encephalitis or encephalopathy associated with reversible lesion of the splenium of the corpus callosum [14]. Transient lesions in the splenium of the corpus callosum can occur in several conditions such as epilepsy, following the sudden withdrawal of antiepileptic drugs, influenza encephalitis, and other conditions such as hemolytic-uremic syndrome [15]. In addition, isolated involvement of the splenium of the corpus callosum may also occur in patients with ADEM [16]. We believe that the lesion in the splenium of the corpus callosum in our patient is demyelinating in nature (likely post-viral).

Children who have ADEM may evolve with motor or cognitive sequela. A recent study described the long-term outcome of 102 children with ADEM [17]. According to this study, $17.2 \%$ of the children had long-term motor or motor and sensory deficit and $8 \%$ had MRC grade 3 or less power in at least one limb.

Potential differential diagnosis in a child with acute leukoencephalomyelopathy includes other viral encephalitides, neuromyelitis optica (NMO), and severe hypoxicischemic injury. Negative rt-PCR for Zika and dengue virus speaks against a co-infection with these endemic viruses in our region and the absence of ophthalmological abnormalities and cardiorespiratory arrest make the possibility of hypoxic-ischemic injury and NMO less likely.

Our patient had neurological symptoms during the acute phase of COVID-19, which reinforces the possibility of a causal relationship between this infection and the CNS involvement. The simultaneous and monophasic brain involvement, mainly in cerebral the white matter and the spinal cord, lack of T1 hypointense lesions, and resolution of the acute MRI findings (restricted diffusion and edema) corroborates a post-infectious demyelinating process such as ADEM. This unique case report highlights the polymorphism of COVID19 neurological manifestations and the possibility of activation of an autoimmune response against the CNS following the SARS-Cov-2 infection.

Funding No funding was received for this study.

\section{Compliance with ethical standards}

Conflict of interest The authors declare that they have no conflict of interest.

Ethical approval All procedures performed in the studies involving human participants were in accordance with the ethical standards of the institutional and/or national research committee and with the 1964 Helsinki Declaration and its later amendments or comparable ethical standards.

Informed consent Informed consent was obtained from all individual participants included in the study.

\section{References}

1. Qiu H, Wu J, Hong L, Luo Y, Song Q, Chen D (2020) Clinical and epidemiological features of 36 children with coronavirus disease 2019 (COVID-19) in Zhejiang, China: an observational cohort study. Lancet Infect Dis 20:689-696

2. Mirzaee SMM, Gonçalves FG, Mohammadifard M, et al (2020) Focal Cerebral arteriopathy in a COVID-19 pediatric patient. Radiology 202197

3. Dugue R, Cay-Martínez KC, Thakur KT, Garcia JA, Chauhan LV, Williams SH, Briese T, Jain K, Foca M, McBrian DK, Bain JM, Lipkin WI, Mishra N (2020) Neurologic manifestations in an infant with COVID-19. Neurology 94:1100-1102

4. de Oliveira FAA, Palmeira DCC, Rocha-Filho PAS (2020) Headache and pleocytosis in CSF associated with COVID-19: case report. Neurol Sci 41:1667

5. Mao L, Jin H, Wang M, Hu Y, Chen S, He Q, Chang J, Hong C, Zhou Y, Wang D, Miao X, Li Y, Hu B (2020) Neurologic 
manifestations of hospitalized patients with coronavirus disease 2019 in Wuhan, China. JAMA Neurol. 77:683-690. https:/doi. org/10.1001/jamaneurol.2020.1127

6. Bridwell R, Long B, Gottlieb M (2020) Neurologic complications of COVID-19. Am J Emerg Med 38:1549.e3-1549.e7

7. Sampaio Rocha-Filho PA, Voss L (2020) Persistent headache and persistent anosmia associated with COVID-19. Headache. 60: 1797-1799. https://doi.org/10.1111/head.13941

8. Zubair AS, McAlpine LS, Gardin T et al (2020) Neuropathogenesis and neurologic manifestations of the coronaviruses in the age of coronavirus disease 2019: a review. JAMA Neurol. 77:10181027. https://doi.org/10.1001/jamaneurol.2020.2065

9. Krupp LB, Tardieu M, Amato MP, Banwell B, Chitnis T, Dale RC, Ghezzi A, Hintzen R, Kornberg A, Pohl D, Rostasy K, Tenembaum S, Wassmer E, for the International Pediatric Multiple Sclerosis Study Group (2013) International Pediatric Multiple Sclerosis Study Group criteria for pediatric multiple sclerosis and immunemediated central nervous system demyelinating disorders: revisions to the 2007 definitions. Mult Scler 19:1261-1267

10. Sáenz-Farret M, Cansino-Torres MA, Sandoval-Rodríguez V et al (2019) The spectrum of acute disseminated encephalomyelitis and mild encephalopathy with reversible splenial lesion. Case Rep Neurol Med 2019:9272074

11. Tenembaum S, Chitnis T, Ness J, Hahn JS, for the International Pediatric MS Study Group (2007) Acute disseminated encephalomyelitis. Neurology 68:S23-S36
12. Tenembaum S, Chamoles N, Fejerman N (2002) Acute disseminated encephalomyelitis: a long-term follow-up study of 84 pediatric patients. Neurology 59:1224-1231

13. Kesselring J, Miller DH, Robb SA et al (1990) Acute disseminated encephalomyelitis. MRI findings and the distinction from multiple sclerosis. Brain 113(Pt 2):291-302

14. Kontzialis M, Soares BP, Huisman TAGM (2017) Lesions in the splenium of the corpus callosum on MRI in children: a review. $\mathrm{J}$ Neuroimaging 27:549-561

15. Park SE, Choi DS, Shin HS, Baek HJ, Choi HC, Kim JE, Choi HY, Park MJ (2017) Splenial lesions of the corpus callosum: disease spectrum and MRI findings. Korean J Radiol 18:710-721

16. Blaauw J, Meiners LC (2020) The splenium of the corpus callosum: embryology, anatomy, function and imaging with pathophysiological hypothesis. Neuroradiology 62:563-585

17. Iype M, Ts A, Kunju PM, Saradakutty G, Sreedharan M, Ahamed SM (2018) Factors related to long term motor, behavioral, and scholastic outcome in children with acute disseminated encephalomyelitis. Pediatr Neurol 89:49-57

Publisher's note Springer Nature remains neutral with regard to jurisdictional claims in published maps and institutional affiliations. 